\title{
Clinical strains of Lactobacillus reduce the filamentation of Candida albicans and protect Galleria mellonella against experimental candidiasis
}

\author{
Rodnei Dennis Rossoni ${ }^{1}$ (D) Marisol dos Santos Velloso ${ }^{1} \cdot$ Lívia Mara Alves Figueiredo $^{1}$ • \\ Carolina Pistille Martins ${ }^{1}$. Antonio Olavo Cardoso Jorge ${ }^{1}$. Juliana Campos Junqueira ${ }^{1}$
}

Received: 31 July 2017 / Accepted: 15 November 2017 / Published online: 23 November 2017

(C) Institute of Microbiology, Academy of Sciences of the Czech Republic, v.v.i. 2017

\begin{abstract}
Candida albicans is the most common human fungal pathogen and can grow as yeast or filaments, depending on the environmental conditions. The filamentous form is of particular interest because it can play a direct role in adherence and pathogenicity. Therefore, the purpose of this study was to evaluate the effects of three clinical strains of Lactobacillus on C. albicans filamentation as well as their probiotic potential in pathogen-host interactions via an experimental candidiasis model study in Galleria mellonella. We used the reference strain Candida albicans ATCC 18804 and three clinical strains of Lactobacillus: L. rhamnosus strain 5.2, L. paracasei strain 20.3, and L. fermentum strain 20.4. First, the capacity of $C$. albicans to form hyphae was tested in vitro through association with the Lactobacillus strains. After that, we verified the ability of these strains to attenuate experimental candidiasis in a Galleria mellonella model through a survival curve assay. Regarding the filamentation assay, a significant reduction in hyphae formation of up to $57 \%$ was observed when C. albicans was incubated in the presence of the Lactobacillus strains, compared to a control group composed of only C. albicans. In addition, when the larvae were pretreated with Lactobacillus spp. prior to C. albicans infection, the survival rate of G. mellonela increased in all experimental groups. We concluded that Lactobacillus influences the growth and expression C. albicans virulence factors, which may interfere with the pathogenicity of these microorganisms.
\end{abstract}

Rodnei Dennis Rossoni dennisrossoni@hotmail.com

Marisol dos Santos Velloso marisol_velloso2@hotmail.com

Lívia Mara Alves Figueiredo livia.figueiredo@fosjc.unesp.br

Carolina Pistille Martins carol.p.martins@hotmail.com

Antonio Olavo Cardoso Jorge olavojorge@fosjc.unesp.br

Juliana Campos Junqueira juliana@fosjc.unesp.br

1 Department of Biosciences and Oral Diagnosis, São Paulo State University (Unesp), Institute of Science and Technology, São José dos Campos, São Paulo, Brazil

\section{Introduction}

Candida albicans is the most common human fungal pathogen and is recognized as the most virulent species of the genus, Candida. This yeast is able to proliferate and invade almost any host tissue (Parahitiyawa et al. 2006; Tsui et al. 2016). This ability of facile invasion is due to a combination of a weakening of the host's immune system and important virulence factors of C. albicans (Höfs et al. 2016). Among the virulence factors of $C$. albicans in superficial and systemic infections is the ability to morphologically transition between yeast and hyphae forms, as well as the ability to form biofilms (Borghi et al. 2016; Egbe et al. 2017; Mayer et al. 2013; Vila et al. 2017).

A biofilm is an assemblage of surface-associated microbial cells that are enclosed in an extracellular, polymeric, substance matrix. Therefore, in C. albicans biofilm development, it is necessary that the hyphae and the basal cell layer formation are attached by an extracellular matrix (Fuchs et al. 2010). The hyphal form is responsible for promoting tissue 
penetration and an escape from immune cells, which leads to the infection process (Tati et al. 2016). The morphological transition from yeast to hyphal filaments occurs in response to a wide variety of conditions in the host environment. These conditions are reproducible in in vitro studies and include the presence of serum, body temperature $\left(37^{\circ} \mathrm{C}\right)$, high $\mathrm{CO}_{2}$ concentration, neutral $\mathrm{pH}$, certain carbon sources or amino acids, and the extracellular matrix of microbial biofilms (de Barros et al. 2017; Kadosh 2016). The inhibition of these virulence factors in C. albicans is an important tool for the treatment of candidiasis.

Recently, certain strains of Lactobacillus have been studied as potential probiotics, which can modulate the immune system and prevent or attenuate experimental candidiasis (Ribeiro et al. 2017; Rossoni et al. 2017). Probiotics are considered to be live microorganisms and can be administered through a wide range of products, including food, medications, and dietary supplements. Various species of microorganisms are known for their probiotic effects, such as Lactobacillus, Bifidobacterium, and Saccharomyces cerevisiae (Alok et al. 2017; Liaskovskii and Podgorskii 2005).

The use of probiotics to inhibit pathogenic microorganisms could be an interesting strategy to control opportunistic infections due to their antagonistic effects on other microorganisms through the release of specific molecules, such as hydrogen peroxide and bacteriocins (Dubovskiy et al. 2013; Peleg et al. 2010). Some Lactobacillus strains can interact with $C$. albicans and seem to inhibit the filamentation of this strain, which results in growth inhibition (Matsubara et al. 2016c; Ribeiro et al. 2017; Smith et al. 2012; Vilela et al. 2015). Despite the great interest in the study and characterization of probiotic strains, the vast majority of these strains originate from the gastrointestinal tract and are called "standard strains"(Matsubara et al. 2016a; Pujia et al. 2017; RiveraEspinoza and Gallardo-Navarro 2010; Verdenelli et al. 2014). In this context, the characterization of clinical strains of Lactobacillus isolated from the oral cavity of our study becomes important and innovative.

Invertebrate models, such as the nematode Caenorhabditis elegans and the insects Drosophila melanogaster and Galleria mellonella, have been developed to study pathogenicity, host-pathogen interaction, and the immune responses resulting from microbial infections (Fedhila et al. 2010). Among these models, the facile inoculum delivery and handling of G. mellonella makes it a desirable model for the study of fungal pathogenesis (Fuchs et al. 2010; Junqueira 2012). Furthermore, the immune system of G. mellonella possesses a number of structural and functional similarities to the innate immune system of mammals (Bergin et al. 2003; Bergin et al. 2006; Mc Namara et al. 2017).

The immune response of $G$. mellonella consists of both cellular and humoral components. The humoral system provides antimicrobial peptides that are released by some cells of G. mellonella. These peptides serve to attack the structures of bacterial and fungal cells that enter the hemolymph. In addition, the resulting cellular response is orchestrated by hemocytes in the antimicrobial process of phagocytosis (Bergin et al. 2006; Wu et al. 2016). In this context, the purpose of this study was to evaluate the in vitro effects of three clinical strains of Lactobacillus on C. albicans filamentation and to verify if these strains are able to increase the survival of $G$. mellonella infected by C. albicans.

\section{Materials and methods}

\section{Strains and growth conditions}

In this study, we used the reference strain Candida albicans ATCC 18804 and three clinical strains of Lactobacillus: $L$. rhamnosus strain 5.2, L paracasei strain 20.3 , and $L$. fermentum strain 20.4. The Lactobacillus strains were isolated from the human oral cavity of an individual without caries, per the approval of the Ethics Committee under protocol 560.479. C. albicans was cultured for $18 \mathrm{~h}$ at $37^{\circ} \mathrm{C}$ in Yeast Nitrogen Base broth (YNB; Difco, Detroit, MI, USA) supplemented with $100 \mathrm{mmol} / \mathrm{L}$ glucose. The Lactobacillus strains were cultured in Lactobacillus Man-Rogosa-Shape broth (MRS broth; Difco, Detroit, USA) for $24 \mathrm{~h}$ at $37{ }^{\circ} \mathrm{C}$ under microaerophilic conditions. The suspension densities were determined with a spectrophotometer (B582, Micronal, Sao Paulo, Brazil) and were then diluted to concentrations of $10^{7}$ and $10^{8}$ cells per $\mathrm{mL}$. The number of cells in the inoculum was confirmed by counting the CFU per $\mathrm{mL}$ following plating on Sabouraud dextrose agar (SDA; Himedia, Mumbai, India) for C. albicans and MRS agar (Difco, Detroit, MI, USA) for Lactobacillus.

\section{In vitro effects of Lactobacillus spp. on C. albicans filamentation}

The filamentation assays were performed in 24-well microtiter plates (TPP®, Trasadingen, Switzerland) following the methodology described by Vilela et al. (2015) with some modifications. In this experiment, the wells were divided into the following groups: C. albicans + PBS (control) and C. albicans + Lactobacillus. One milliliter of distilled water supplemented with $10 \%$ fetal bovine serum and $100 \mathrm{~mL}$ of a C. albicans suspension $\left(10^{7}\right.$ cells per $\mathrm{mL}$ ) were added to each well. Then, $50 \mu \mathrm{L}$ of Lactobacillus cells $\left(10^{7}\right.$ cells per $\left.\mathrm{mL}\right)$ was added to each well. In the control group, $50 \mu \mathrm{L}$ of PBS was added instead of Lactobacillus. The plates were incubated at $37{ }^{\circ} \mathrm{C}$ for $24 \mathrm{~h}$. Afterwards, $50 \mu \mathrm{L}$ of the culture contained in each well was spread onto a glass slide and observed 
under a light microscope at $40 \times$ magnification. The images were analyzed in terms of $C$. albicans morphology and hyphae quantification, which were determined through examining 10 microscopic fields per slide. According to Vilela et al. (2015), the following scores were assigned for the number of hyphae present in each microscopic field: score 0: no hyphae; score 1: 1 to 3 hyphae; score 2: 4 to 10 hyphae; score 3: 11 to 20 hyphae; and score 4: more than 20 hyphae. The filamentation percentage was calculated by considering the total amount of hyphae in a microscopy field of the C. albicans control group in relation to the groups associated with Lactobacillus. The filamentation assay was performed as three independent experiments with $n=5$ wells per group.

\section{In vivo effects of Lactobacillus spp. on G. mellonella survival}

In this study, the methodology described by Vilela et al. (2015) was adhered to, with some modifications. $G$. mellonella in the final larval stage and with similar weights and sizes of 200-250 mg were randomly selected and divided into seven experimental groups: one negative control group (no injection), one positive control group (PBS injection), two positive control groups (C. albicans injection + PBS and PBS + C. albicans groups), two "prophylactic" groups (Lactobacillus and C. albicans injections), and one "therapeutic" group (C. albicans and Lactobacillus injections). For the prophylactic groups, we had two different groups: prophylactic provisions of Lactobacillus $24 \mathrm{~h}$ prior to C. albicans infection (Lactobacillus $1 \times$ ) and prophylactic provisions of Lactobacillus 48 and $24 \mathrm{~h}$ prior to $C$. albicans infection (Lactobacillus $2 \times$ ). For the therapeutic group, we injected Lactobacillus after $1 \mathrm{~h}$ of C. albicans infection.

Previously, our group determined the sub-lethal inoculum concentration of these Lactobacillus strains by injecting larvae with serial dilutions of the bacteria. Based on the results of Rossoni et al. (2017), a concentration of $10^{6}$ cells per larva was adopted for all subsequent assays, since it is the same concentration that is typically used for C. albicans infections.

To evaluate the effects of probiotics on C. albicans infections, the larvae were injected with Lactobacillus and $C$. albicans, according to the previously described experimental groups, through the last left proleg (volume of $10 \mu \mathrm{L}$ ). Larvae were incubated at $37{ }^{\circ} \mathrm{C}$ and monitored daily for survival. The larvae were considered to be dead when they did not present any movement in response to touch. The death of all larvae in the experimental group or the transition to a pupal form was determined at the end of the experiment. The survival curve was performed in two independent experiments using 16 larvae per group.

\section{Statistical analysis}

A statistical analysis was performed using GraphPad Prism 6 software (GraphPad Software, Inc., La Jolla, CA, USA). The scores obtained through in vitro filamentation analysis were compared by using Kruskal-Wallis and Dunn's tests. A Student's $t$ test was used for filamentation percentage calculations. The survival curves were plotted, and statistical analysis was performed via a Kaplan-Meier test. A $p$ value $\leq 0.05$ was considered significant.

\section{Results}

\section{In vitro effects of Lactobacillus spp. on C. albicans filamentation}

In the filamentation assay, we observed a large number of $C$. albicans hyphae in the control groups with PBS. However, we verified that hyphae formation was significantly inhibited when $C$. albicans was incubated in the presence of Lactobacillus strains $5.2(p=0.0004)$ and $20.4(p=0.0327)$ compared to the control group. L. paracasei 20.3 was able to reduce $C$. albicans filamentation, but the difference was not statistically significant compared to the control group (Fig. 1).

The best reduction in C. albicans filamentation was observed in its interaction with L. rhamnosus 5.2 (Fig. 2). More specifically, the results obtained from the $C$. albicans control group were compared with heterotypic groups; we found a $57 \%$ reduction in germination values when this strain was associated with $C$. albicans, as opposed to $31 \%$ with $L$. fermentum 20.4 and $17 \%$ for L. paracasei 20.3 (Fig. 3).

\section{In vivo effects of Lactobacillus spp. on G. mellonella survival}

In the in vivo assay, we found that $100 \%$ of larvae survived in the PBS control groups, demonstrating that there was no interference due to the particular batch of larvae used or the handling technique used by the manipulator. In the positive control group, the infection with $C$. albicans without previously injecting Lactobacillus strains caused death in $100 \%$ of the larvae within $24 \mathrm{~h}$.

When the larvae were pretreated with Lactobacillus spp. prior to C. albicans infection, the survival rate of G. mellonela increased in all experimental groups. Highlighting the experimental groups involving a 48-h "prophylactic" interaction (Lactobacillus inoculated 24 and $48 \mathrm{~h}$ prior to C. albicans infection), larvae survival increased up to $80 \%$ with $L$. rhamnosus injection (Fig. 4a), 20\% with $L$. paracasei injection (Fig. 4b), and 60\% with L. fermentum injection (Fig. 4c). In agreement with the results obtained in the in vitro 
Fig. 1 a Median scores obtained by determining the number of hyphae in the in vitro C. albicans filamentation assay for the following groups: C. albicans control group and cells of $L$. rhamnosus $5.2+$ C. albicans group. b Median scores obtained by determining the number of hyphae in the in vitro $C$. albicans filamentation assay for the following groups: C. albicans control group and cells of $L$. paracasei $20.3+$ C. albicans group. c Median scores obtained by determining the number of hyphae in the in vitro C. albicans filamentation assay for the following groups: C. albicans control group and cells of $L$. fermentum $20.4+$ C. albicans group. A significant hyphae reduction was observed in the interactions with L. rhamnosus $(p=0.0004)$ and L. fermentum ( $p=0.0327$ ) compared to the $C$. albicans control group (MannWhitney test, $p \leq 0.05$ )
Fig. 2 Light microscopy photomicrographs of in vitro Candida albicans filamentation. a Control group (PBS): intense formation of hyphae. $\mathbf{b} L$. rhamnosus $5.2+$ C. albicans group: reduction of hyphae formation. Original magnification: $\times 400$ a
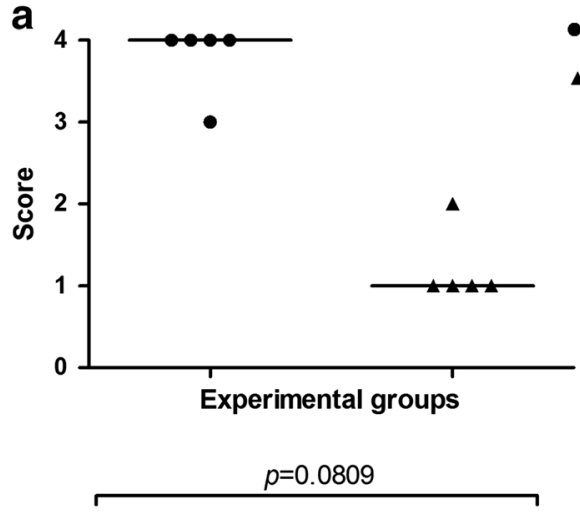

b

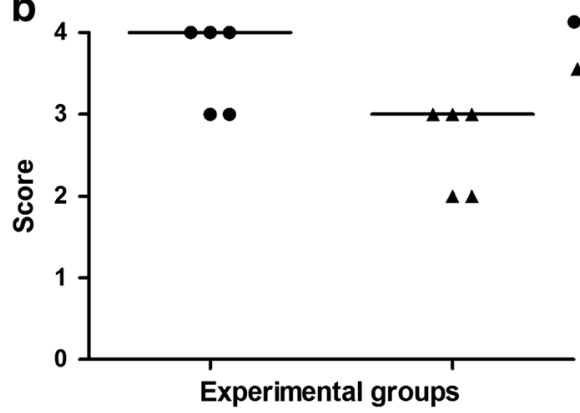

Experimental groups

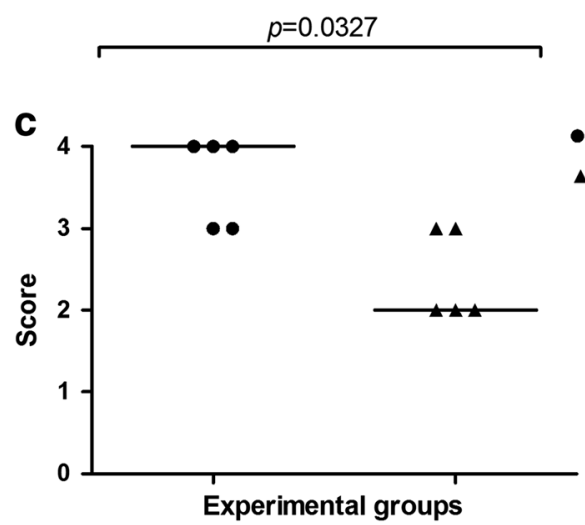

- PBS + C. albicans (Control)

- Cells of $L$. rhamnosus $5.2+C$. albicans
- PBS + C. albicans (Control)

- Cells of $L$. paracasei $20.3+C$. albicans
- PBS + C. albicans (Control)

- Cells of $L$. fermentum $20.4+C$. albicans
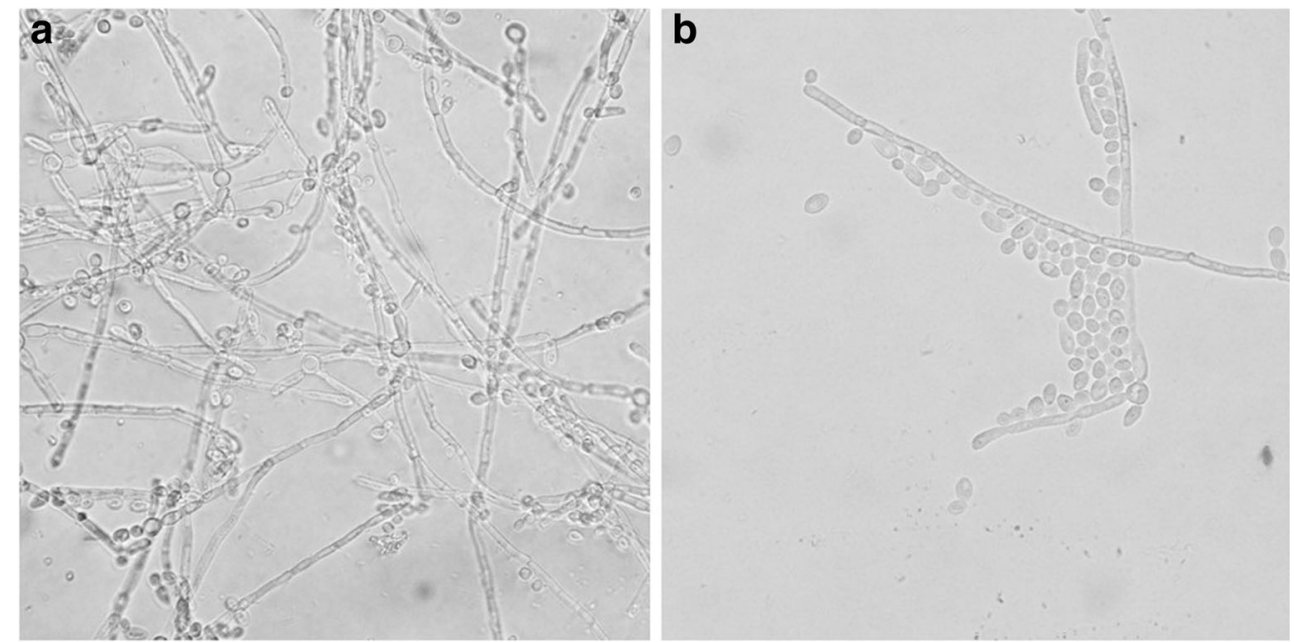
Fig. 3 Lactobacillus strains reduce the $C$. albicans germination percentage. Percentage of germination, expressed as the mean values of hyphae and pseudohyphae of $C$. albicans alone (control group) and when associated with Lactobacillus spp. A significant hyphae reduction was observed in the interaction with $L$. rhamnosus $(p=0.0005), L$. paracasei $(p=$ 0.0395 ), and L. fermentum ( $p=$ $0.0113)$ compared to the $C$. albicans control group ( $t$ test, $p \leq$ 0.05)

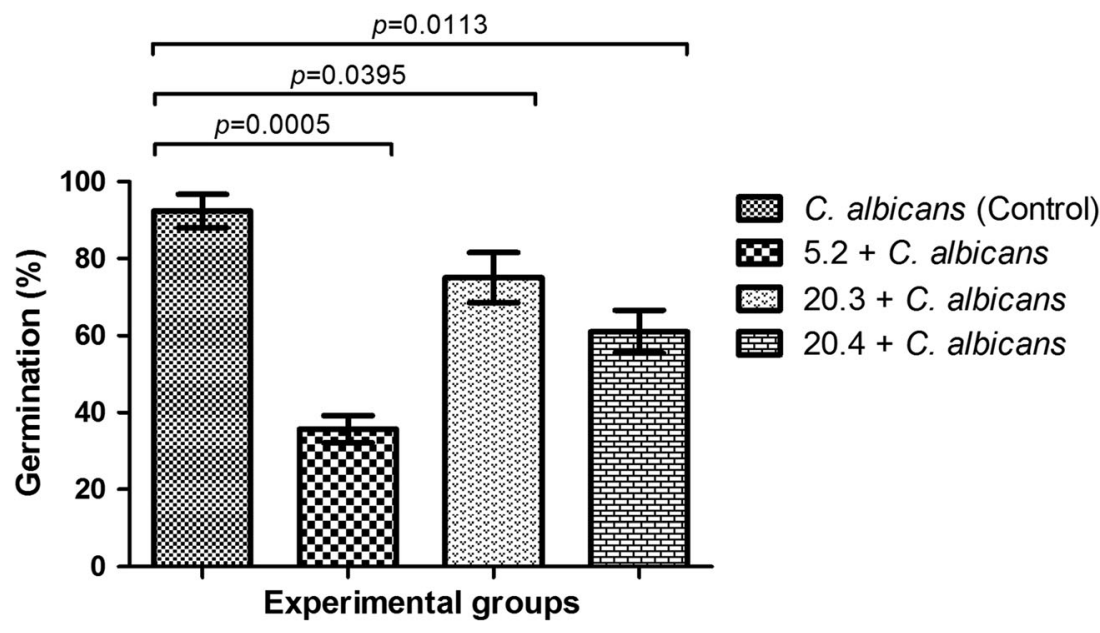

filamentation assays, L. rhamnosus promoted the greatest survival rate of $G$. mellonella compared to the other strains.

\section{Discussion}

In this study, we evaluated the inhibitory potential of Lactobacillus on $C$. albicans filamentation and whether this could affect the in vivo survival of larvae infected with $C$. albicans. These data agree with Matsubara et al. (2016c), who evaluated the inhibitory effects of the L. rhamnosus LR32 to the Candida biofilm and filamentation in vitro. In the presence of probiotic, the biofilm had a predominance of yeast instead of hyphae, while the control biofilm presented a denser distribution of filamentous cells, showing that the direct contact of probiotic cells with $C$. albicans biofilms was essential for the anti-filamentation effect. Various in vitro studies have demonstrated the antifungal effect of probiotics against $C$. albicans isolates from the oral cavity and gastrointestinal tract showing the importance of adjuvant therapies since there are no adverse effects directly related to probiotics (Coman et al. 2014; Hasslof et al. 2010; Matsubara et al. 2016b; Verdenelli et al. 2014).

An analysis of in vitro filamentation showed that the interaction between our clinical strains of Lactobacillus and $C$. albicans reduced the number of hyphae compared to the $C$. albicans control group. The largest reduction in C. albicans filamentation was observed through its interaction with $L$. rhamonsus 5.2, in which the median filamentation score of C. albicans was reduced by $57 \%$. One hypothesis regarding our results is that the strain, L. rhamnosus 5.2, can directly act against the virulence of $C$. albicans through producing some bacteriocins, lactic acid, or other metabolites capable of reducing the number of hyphae. In this context, the influence of these strains with probiotic potential on $C$. albicans hyphae formation suggests new study directions, such as extracting and isolating the molecules released in the metabolism of
Lactobacillus. The results also support new therapeutic protocols against candidiasis.

In agreement with the results obtained here, Leão et al. (2015) evaluated the influence of L. rhamnosus ATCC 1465 on the expression of $C$. albicans virulence factors in vitro. A suspension of L. rhamnosus was cultured, and its influence on $C$. albicans biofilm and germ tube formation was evaluated after 24, 48, and $72 \mathrm{~h}$ of Lactobacillus-Candida interaction. The authors found a significant reduction in $C$. albicans growth and germ tube formation at all evaluated time points, suggesting that L. rhamnosus may interfere with the pathogenicity of $C$. albicans. In addition, de Oliveira et al. (2017) related the probiotic activity of $L$. rhamnosus ATCC 7469 in mouse macrophages and in $G$. mellonella model. The authors verified that L. rhamnosus was able to stimulate the tumor necrosis factor (TNF)- $\alpha$ and interleukin IL-1 $\beta$, IL-6, and IL-17 production, and this immune modulation may be related to the decreased $C$. albicans infection in G. mellonella.

Lactobacillus is one of the most common bacteria used as a probiotic. Probiotics are bacteria that provide beneficial effects to the host. They are used in the prevention and treatment of diseases, in the regulation of the intestinal microbiota, and as immunostimulants and are able to compete directly with other microorganisms for adhesion to the host epithelium (Hashemi et al. 2016; Patel and DuPont 2015). Numerous studies have shown that Lactobacillus species have acidogenic capacities and acid tolerances that can directly affect the formation of hyphae (Badet and Thebaud 2008; Bandara et al. 2017; Matsubara et al. 2016b; Matsubara et al. 2016c; Ribeiro et al. 2017). According Mayer et al. (2013), a range of environmental cues are able to affect $C$. albicans morphology. For example, at a low $\mathrm{pH}(<6), C$. albicans cells predominantly grow in the yeast form, while at a high $\mathrm{pH}(>7)$, hyphal growth is induced. These data suggest that the Lactobacillus strains in this study acidify the $\mathrm{pH}$ of the medium to a point that is able to reduce the formation of hyphae. 

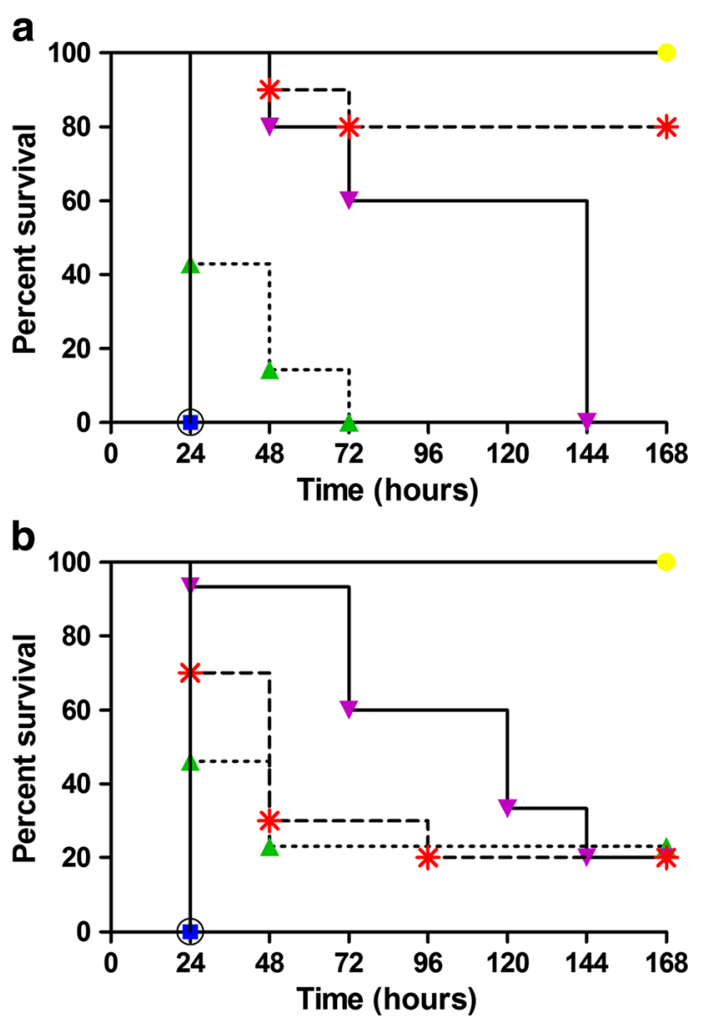
- A- Controls
$\rightarrow$ B- C. albicans + PBS
-A- C- C. albicans + L. rhamnosus (therapeutic group $1 \mathrm{~h}$ )
$\rightarrow$ D- L. rhamnosus $1 \mathrm{x}+$ C.albicans (prophylactic group 24h)
*- E- L. rhamnosus $2 \mathrm{x}+$ C. albicans (prophylactic group $24 \mathrm{~h}$ and $48 \mathrm{~h}$ )
$\ominus$ F-PBS + C.albicans

\author{
- A- Controls \\ $\rightarrow$ B- C. albicans + PBS \\ - C- C. albicans + L. paracasei (therapeutic group) \\ $\rightarrow$ D- L. paracasei $1 \mathrm{x}+$ C.albicans (prophylactic group $24 \mathrm{~h}$ ) \\ *- E- L. paracasei $2 \mathrm{x}+\mathrm{C}$. albicans (prophylactic group $24 \mathrm{~h}$ and $48 \mathrm{~h}$ ) \\ $\ominus$ F- PBS + C.albicans
}

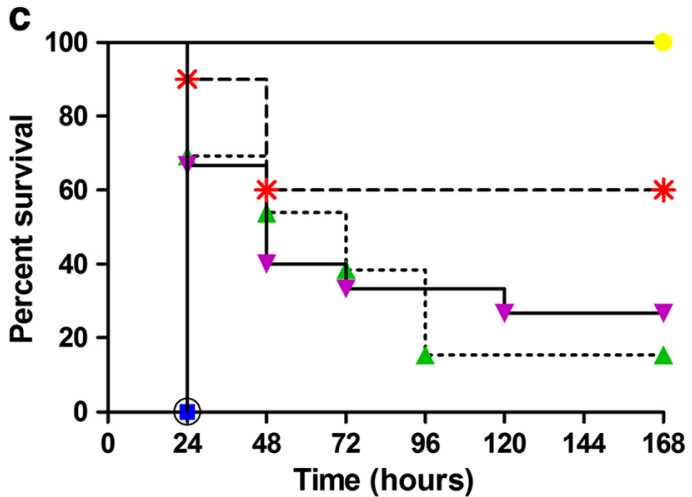

Fig. 4 Lactobacillus spp. prolongs the survival of G. mellonella larvae infected with $C$. albicans. There was a significant difference between the "Lactobacillus strain + C. albicans group" and "PBS + C. albicans control group." a L. rhamnosus 5.2: therapeutic group (C. albicans + L. rhamnosus $/ C$. albicans + PBS) $p=0.0269$; prophylactic group $24 \mathrm{~h}$ $(\mathrm{PBS}+C$. albicans $/$ L. rhamnosus $1 \times+C$. albicans $) p=0.0001$; prophylactic group 24 and $48 \mathrm{~h}(\mathrm{PBS}+C$. albicans/L. rhamnosus $2 \times+$ C. albicans $) p=0.0001$. b $L$. paracasei 20.3: therapeutic group $(C$. albicans + L. paracasei/C. albicans + PBS) $p=0.0036$; prophylactic

Based on the in vitro data, we expanded this study to include an in vivo model of experimental candidiasis, using $G$. mellonella. Larvae survival increased in all groups associated with Lactobacillus strains, especially for the 48-h "prophylactic" groups (prophylactic inoculation of Lactobacillus at 24 and $48 \mathrm{~h}$ prior to Candida infection). In these groups, larvae survival increased up to $80 \%$ with an L. rhamnosus injection, $20 \%$ with an $L$. paracasei injection, and $60 \%$ with an L. fermentum injection, compared with the $C$. albicans control group. group $24 \mathrm{~h}(\mathrm{PBS}+C$. albicans $/$. paracasei $1 \times+C$. albicans $) p=$ 0.0001 ; prophylactic group 24 and $48 \mathrm{~h}(\mathrm{PBS}+C$. albicans $/ L$. paracasei $2 \times+C$. albicans $) p=0.0001$. c L. fermentum 20.4: therapeutic group $(C$. albicans $+L$. fermentum $/ C$. albicans + PBS $) p=$ 0.0001 ; prophylactic group $24 \mathrm{~h}(\mathrm{PBS}+C$. albicans/L. fermentum $1 \times+$ C. albicans) $p=0.0001$; prophylactic group 24 and $48 \mathrm{~h}(\mathrm{PBS}+C$. albicans $/ L$. fermentum $2 \times+C$. albicans) $p=0.0001$. Controls are composed of groups that received only Lactobacillus or PBS and by the no injection group. Kaplan-Meier test, $p \leq 0.05$

L. rhamnosus 5.2 was the strain of Lactobacillus with the greatest probiotic activity promoting larvae survival, as well as the strain that further reduced the formation of hyphae in vitro. This indicates that this Lactobacillus strain is capable of altering the morphogenesis of C. albicans, influencing larvae survival. A concurring study by Borghi et al. (2014) investigated whether biofilm production by $C$. albicans clinical isolates could be a hallmark of virulence in G. mellonella. The authors found a positive correlation between the biofilm 
producing strains and the virulence of $G$. mellonella due to the capacity of $C$. albicans to produce filaments and form a biofilm structure inside the larvae.

Recently, members of our research group developed a study to evaluate the immunomodulatory action of the strain, L. paracasei 28.4, in G. mellonella experimental candidiasis. Rossoni et al. (2017) showed that prior exposure to a dose of L. paracasei 28.4 activates the G. mellonella immune system, which may allow the larvae to combat a lethal infection by $C$. albicans. This effect was mediated by an increase in circulating hemocytes and the production of elevated levels of antimicrobial peptides that consequently reduce Candida cell numbers in G. mellonella hemolymph. These data reinforce the prophylactic results of our study, in which we observed up to an $80 \%$ increase in larval survival.

In our study, we also observed that the therapeutic groups treated with Lactobacillus strains were able to increase larval survival, but this rate was much lower compared to that in the prophylactic groups (0 to 23\%). With these data, we can suggest that Lactobacillus needs more time inside the larvae to produce metabolites that affect the pathogenicity of $C$. albicans, as highlighted by the times used for the prophylactic groups (24 and $48 \mathrm{~h}$ ). In addition, when lactobacilli were injected into the hemolymph of the larvae in the therapeutic groups, these insects were already infected by $C$. albicans. This factor may have made it more difficult to attenuate the fungal infection.

In this context, more studies should be performed in order to reveal the exact mechanisms used by lactobacilli in the inhibition of Candida infections, for a better understanding of this disease in humans, and to find alternative therapies that do not involve the use of drugs. We concluded that Lactobacillus influences the growth of C. albicans and expression of its virulence factors, which may interfere with the pathogenicity of this microorganism. The prophylactic groups of all lactobacilli used in this study resulted in better survival curves compared to the therapeutic group.

Funding This study was supported by the São Paulo Council of Research-FAPESP, Brazil (Grants 2013/25181-8 and 2015/09770-9).

Compliance with ethical standards This study has been approved by the Ethics Committee of São Paulo State University (Unesp) under protocol 560.479 .

Conflict of interest The authors declare that they have no conflict of interest.

\section{References}

Alok A, Singh ID, Singh S, Kishore M, Jha PC, Iqubal MA (2017) Probiotics: a new era of biotherapy. Adv Biomed Res 6(1):31. https://doi.org/10.4103/2277-9175.192625
Badet C, Thebaud NB (2008) Ecology of lactobacilli in the oral cavity: a review of literature. Open Microbiol J 2(1):38-48. https://doi.org/ $10.2174 / 1874285800802010038$

Bandara HM, Matsubara VH, Samaranayake LP (2017) Future therapies targeted towards eliminating Candida biofilms and associated infections. Expert Rev Anti-Infect Ther 15(3):299-318. https://doi.org/ 10.1080/14787210.2017.1268530

Bergin D, Brennan M, Kavanagh K (2003) Fluctuations in haemocyte density and microbial load may be used as indicators of fungal pathogenicity in larvae of Galleria mellonella. Microbes Infect 5(15):1389-1395. https://doi.org/10.1016/j.micinf.2003.09.019

Bergin D, Murphy L, Keenan J, Clynes M, Kavanagh K (2006) Preexposure to yeast protects larvae of Galleria mellonella from a subsequent lethal infection by Candida albicans and is mediated by the increased expression of antimicrobial peptides. Microbes Infect 8(8):2105-2112. https://doi.org/10.1016/j.micinf.2006.03.005

Borghi E, Romagnoli S, Fuchs BB, Cirasola D, Perdoni F, Tosi D, Braidotti P, Bulfamante G, Morace G, Mylonakis E (2014) Correlation between Candida albicans biofilm formation and invasion of the invertebrate host Galleria mellonella. Future Microbiol 9(2):163-173. https://doi.org/10.2217/fmb.13.159

Borghi E, Borgo F, Morace G (2016) Fungal biofilms: update on resistance. Adv Exp Med Biol 931:37-47. https://doi.org/10.1007/5584 2016 7

Coman MM, Verdenelli MC, Cecchini C, Silvi S, Orpianesi C, Boyko N, Cresci A (2014) In vitro evaluation of antimicrobial activity of Lactobacillus rhamnosus IMC 501((R)), Lactobacillus paracasei IMC 502((R)) and SYNBIO((R)) against pathogens. J Appl Microbiol 117(2):518-527. https://doi.org/10.1111/jam.12544

de Barros PP, Freire F, Rossoni RD, Junqueira JC, Jorge AOC (2017) Candida krusei and Candida glabrata reduce the filamentation of Candida albicans by downregulating expression of HWP1 gene. Folia Microbiol (Praha) 62(4):317-323. https://doi.org/10.1007/ s12223-017-0500-4

de Oliveira FE, Rossoni RD, de Barros PP, Begnini BE, Junqueira JC, Jorge AOC, Leão MVP, de Oliveira LD (2017) Immunomodulatory effects and anti-Candida activity of lactobacilli in macrophages and in invertebrate model of Galleria mellonella. Microb Pathog 110: 603-611. https://doi.org/10.1016/j.micpath.2017.08.006

Dubovskiy IM, Whitten MMA, Yaroslavtseva ON, Greig C, Kryukov VY, Grizanova EV, Mukherjee K, Vilcinskas A, Glupov VV, Butt TM (2013) Can insects develop resistance to insect pathogenic fungi? PLoS One 8(4):e60248. https://doi.org/10.1371/journal.pone. 0060248

Egbe NE, Dornelles TO, Paget CM, Castelli LM, Ashe MP (2017) Farnesol inhibits translation to limit growth and filamentation in C. albicans and S. cerevisiae. Microb Cell 4(9):294-304. 10. 15698/mic2017.09.589

Fedhila S, Buisson C, Dussurget O, Serror P, Glomski IJ, Liehl P, Lereclus D, Nielsen-LeRoux C (2010) Comparative analysis of the virulence of invertebrate and mammalian pathogenic bacteria in the oral insect infection model Galleria mellonella. J Invertebr Pathol 103(1):24-29. https://doi.org/10.1016/j.jip.2009.09.005

Fuchs BB, Eby J, Nobile CJ, El Khoury JB, Mitchell AP, Mylonakis E (2010) Role of filamentation in Galleria mellonella killing by Candida albicans. Microbes Infect 12(6):488-496. https://doi.org/ 10.1016/j.micinf.2010.03.001

Hashemi A, Villa CR, Comelli EM (2016) Probiotics in early life: a preventative and treatment approach. Food Funct 7(4):1752-1768. https://doi.org/10.1039/c5fo01148e

Hasslof P, Hedberg M, Twetman S, Stecksen-Blicks C (2010) Growth inhibition of oral mutans streptococci and candida by commercial probiotic lactobacilli - an in vitro study. BMC Oral Health 10(1):18. https://doi.org/10.1186/1472-6831-10-18

Höfs S, Mogavero S, Hube B (2016) Interaction of Candida albicans with host cells: virulence factors, host defense, escape strategies, 
and the microbiota. J Microbiol 54(3):149-169. https://doi.org/10. 1007/s12275-016-5514-0

Junqueira JC (2012) Galleria mellonella as a model host for human pathogens: recent studies and new perspectives. Virulence 3(6): 474-476. https://doi.org/10.4161/viru.22493

Kadosh D (2016) Control of Candida albicans morphology and pathogenicity by post-transcriptional mechanisms. Cell Mol Life Sci 73(22):4265-4278. https://doi.org/10.1007/s00018-016-2294-y

Leao MV, Goncalves Silva CR, Santos SS, Leite PG (2015) Lactobacillus rhamnosus may change the virulence of Candida albicans. Rev Bras Ginecol Obstet 37:417-420. https://doi.org/10.1590/SO100720320150005217

Liaskovskii TM, Podgorskii VS (2005) Assessment of probiotics according to the international organizations (FAO/WHO). Mikrobiol $\mathrm{Z}$ 67(6):104-112

Matsubara VH, Bandara HM, Ishikawa KH, Mayer MP, Samaranayake LP (2016a) The role of probiotic bacteria in managing periodontal disease: a systematic review. Expert Rev Anti-Infect Ther 14(7): 643-655. https://doi.org/10.1080/14787210.2016.1194198

Matsubara VH, Bandara HM, Mayer MP, Samaranayake LP (2016b) Probiotics as antifungals in mucosal candidiasis. Clin Infect Dis 62(9):1143-1153. https://doi.org/10.1093/cid/ciw038

Matsubara VH, Wang Y, Bandara HM, Mayer MP, Samaranayake LP (2016c) Probiotic lactobacilli inhibit early stages of Candida albicans biofilm development by reducing their growth, cell adhesion, and filamentation. Appl Microbiol Biotechnol 100(14):64156426. https://doi.org/10.1007/s00253-016-7527-3

Mayer FL, Wilson D, Hube B (2013) Candida albicans pathogenicity mechanisms. Virulence 4(2):119-128. https://doi.org/10.4161/viru. 22913

Mc Namara L, Carolan JC, Griffin CT, Fitzpatrick D, Kavanagh K (2017) The effect of entomopathogenic fungal culture filtrate on the immune response of the greater wax moth, Galleria mellonella. J Insect Physiol 100:82-92. https://doi.org/10.1016/j.jinsphys.2017. 05.009

Parahitiyawa NB et al (2006) Interspecies variation in Candida biofilm formation studied using the Calgary biofilm device. APMIS 114(4): 298-306. https://doi.org/10.1111/j.1600-0463.2006.apm_394.x

Patel R, DuPont HL (2015) New approaches for bacteriotherapy: prebiotics, new-generation probiotics, and synbiotics. Clin Infect Dis 60(Suppl 2):S108-S121. https://doi.org/10.1093/cid/civ177

Peleg AY, Hogan DA, Mylonakis E (2010) Medically important bacterialfungal interactions. Nat Rev Microbiol 8(5):340-349. https://doi. org/10.1038/nrmicro2313
Pujia AM, Costacurta M, Fortunato L, Merra G, Cascapera S, Calvani M, Gratteri S (2017) The probiotics in dentistry: a narrative review. Eur Rev Med Pharmacol Sci 21(6):1405-1412

Ribeiro FC, de Barros PP, Rossoni RD, Junqueira JC, Jorge AO (2017) Lactobacillus rhamnosus inhibits Candida albicans virulence factors in vitro and modulates immune system in Galleria mellonella. J Appl Microbiol 122(1):201-211. https://doi.org/10.1111/jam.13324

Rivera-Espinoza Y, Gallardo-Navarro Y (2010) Non-dairy probiotic products. Food Microbiol 27(1):1-11. https://doi.org/10.1016/j.fm. 2008.06.008

Rossoni RD, Fuchs BB, de Barros PP, Velloso MD, Jorge AO, Junqueira JC, Mylonakis E (2017) Lactobacillus paracasei modulates the immune system of Galleria mellonella and protects against Candida albicans infection. PLoS One 12(3):e0173332. https://doi.org/10. 1371/journal.pone.0173332

Smith AR, Macfarlane GT, Reynolds N, O'May GA, Bahrami B, Macfarlane S (2012) Effect of a synbiotic on microbial community structure in a continuous culture model of the gastric microbiota in enteral nutrition patients. FEMS Microbiol Ecol 80(1):135-145. https://doi.org/10.1111/j.1574-6941.2011.01279.x

Tati S, Davidow P, McCall A, Hwang-Wong E, Rojas IG, Cormack B, Edgerton M (2016) Candida glabrata binding to Candida albicans hyphae enables its development in oropharyngeal candidiasis. PLoS Pathog 12(3):e1005522. https://doi.org/10.1371/journal.ppat. 1005522

Tsui C, Kong EF, Jabra-Rizk MA (2016) Pathogenesis of Candida albicans biofilm. Pathog Dis 74(4):ftw018. https://doi.org/10. 1093/femspd/ftw018

Verdenelli MC, Coman MM, Cecchini C, Silvi S, Orpianesi C, Cresci A (2014) Evaluation of antipathogenic activity and adherence properties of human Lactobacillus strains for vaginal formulations. J Appl Microbiol 116(5):1297-1307. https://doi.org/10.1111/jam.12459

Vila T, Romo JA, Pierce CG, McHardy SF, Saville SP, Lopez-Ribot JL (2017) Targeting Candida albicans filamentation for antifungal drug development. Virulence 8(2):150-158. https://doi.org/10.1080/ 21505594.2016.1197444

Vilela SF et al (2015) Lactobacillus acidophilus ATCC 4356 inhibits biofilm formation by $C$. albicans and attenuates the experimental candidiasis in Galleria mellonella. Virulence 6(1):29-39. https:// doi.org/10.4161/21505594.2014.981486

Wu G, Xu L, Yi Y (2016) Galleria mellonella larvae are capable of sensing the extent of priming agent and mounting proportionatal cellular and humoral immune responses. Immunol Lett 174:45-52. https://doi.org/10.1016/j.imlet.2016.04.013 